\title{
Challenges and Opportunities in Transmission Electron Microscopy for Revealing the Fate of Inorganic Nanomaterials in Living Beings
}

\author{
Damien Alloyeau ${ }^{1}$, Jeanne Volatron ${ }^{2}$, Yasir Javed ${ }^{1}$, Max Piffoux ${ }^{2}$, Walid Dachraoui ${ }^{1}$, Dan Elgrabli ${ }^{2}$, \\ Lenaic Lartigue $^{1}$, Florence Gazeau ${ }^{1}$, Christian Ricolleau ${ }^{1}$. \\ 1. Laboratoire Matériaux et Phénomènes Quantiques, CNRS - Université Paris-Diderot, Paris, France \\ 2. Laboratoire Matière et Systèmes Complexes, CNRS - Université Paris-Diderot, Paris, France
}

The development of safe and efficient nanomaterials for biomedicine requires understanding their life cycle in the body. In that regards, the multi-functionalities of transmission electron microscopy (TEM) provide the unique opportunity to study at the atomic scale what happens to nanomaterials when they are injected in vitro and in vivo. Over the last 8 years, we have exploited TEM to reveal the biodistribution, the biodegradation and recycling processes of many promising nanomaterials for biomedical applications, including magnetic (Fe and Co nano-oxides), plasmonic (gold) and graphitic (nanotubes) nanostructures [1-7]. Through an overview of these studies, we want to highlight the possibilities and challenges in TEM for revealing the fate of inorganic nanomaterials in the organism.

The first question that arises when administrating nanomaterials to a living being is where are they going? Ex vivo TEM analyses together with global magnetic and elementary analyses allows revealing the biodistribution of nanomaterials at the tissue and sub-cellular levels. With this multiscale approach, we demonstrated that the journey of nanomaterials injected in mice mainly ends the liver and spleen inside intracellular lysosomes of macrophage cells (Fig. 1a) [1-2]. Nevertheless, we also evidenced an elementspecific biodistribution, since the iron oxide and gold portions of nanodimers, although they are injected within the same nanostructures, are preferentially uptaken by the liver and the spleen, respectively [4].

The biodegradation of nanomaterials in cellular media is of primary importance for both material scientists interested in the modifications of nanostructures that could affect their potential for medical applications, and biologists anxious to know the biotransformation processes and resulting products of degradation, so as to assess their potential toxicity. We have shown that metal, oxide, or graphitic nanomaterials slowly degrade via oxidative-etching processes driven by cells (Fig. 1a), [1-5] but their biopersistence is also compositiondependent. For example, iron-oxide nanoparticles fully dissolved in a few months while gold NPs slowly reduced in size but are still observed up to 18 months after injection [4].

Interestingly, the relation between the atomic-scale structure and degradability of nanomaterials can be deeply studied by working in model media mimicking the cellular environment. We can then exploit in situ TEM approaches, including step-by-step HRTEM analyses [2-4], or real-time observations by liquid-cell TEM (Fig. 1b) [5], to monitor the corrosion processes of individual nanostructures over time. In a general manner, the degradation mechanisms of nanostructures is governed by the presence of defects and more importantly, by the access of chelating agents to the nanocrystal surface. Such mechanistic insights into nanomaterial degradation allow establishing relevant strategy to modulate their life-cycle in the body. In line with in vivo investigations, we notably demonstrated that biodegradation speed of iron-oxide nanoparticles depends on the nature and distribution of the coating layer over the nanocrystals and it can be drastically slow-down with full shells of amphiphilic polymers or gold that act as bio-persistent nano-shields [2-4].

Intra-cellular degradation of nanomaterials raises concerns about the ability of cells to store and to recycle potentially toxic metal ions. Ex vivo HRTEM and STEM EDX analyses unambiguously revealed the 
presence of iron-rich ferritin proteins at the vicinity of degraded iron-oxide nanostructures $[1,2]$. This crucial result suggests the transfer of iron from the injected nanocrystals to the ferritin nanocavities. More recently, we also demonstrated both in model media and in vivo, the capacity of ferritin cages to store the degradation products of cobalt-ferrite NPs and even to regulate their degradation rate $[7,8]$. This transfer of different metals into ferritins is certainly a quintessential process in the local degradation of nanomaterials and recycling of their by-products.

Visualizing nanomaterials / biomaterials interactions directly in physiological media would be a decisive step forward to elucidate the way taken by cells to process nanomaterials. However, before meeting this challenge, we must determine if the dynamics of biomaterials can be studied with liquid-cell TEM and evaluate the inevitable electron-beam effects on biological processes. In that regards, we will show encouraging results obtained on extra-cellular vesicles [9]. By monitoring the dynamics of these biomaterials, including their aggregation, fusion and deformation in response to osmotic stress, our study paves the way towards promising applications of liquid-cell TEM for examining the dynamics of nanomaterials in complex biological conditions.

\section{References:}

[1] Levy et al. Biomaterials 32 (2011), p. 3988.

[2] Lartigue et al. ACS nano 7 (2013), p. 3939.

[3] Javed et al. Small 10 (2014), p. 3325.

[4] Kolosnjaj-Tabi et al. ACS Nano 9 (2015), p. 7925.

[5] Elgrabli et al. ACS Nano 9 (2015), p. 10113.

[6] N. Feliu et al, Chemical Society Reviews 45 (2016), p. 2440.

[7] Volatron et al. Scientific Report 7 (2017), p. 40075.

[8] Volatron et al. Small 13 (2017), p. 1602030.

[9] Piffoux et al. Nanoscale 10 (2018), p. 1234.

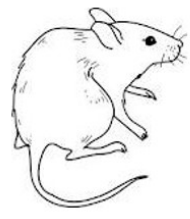

In Vivo

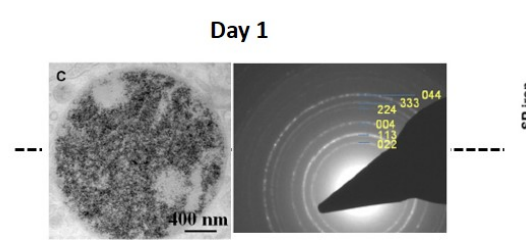

Iron oxide NPs in Intra-cellular lysosome of macrophages of the Liver

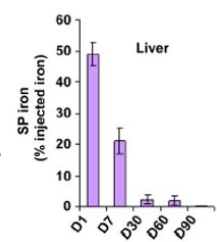

Significant decrease of the superparamagnetic iron content due to the degradation of the atomic structure

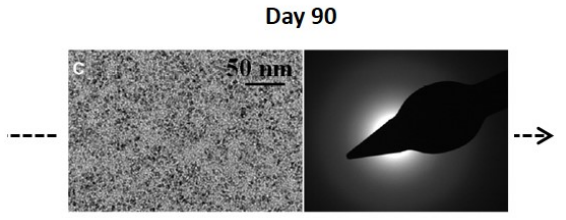

b

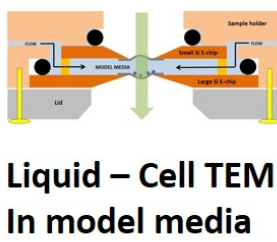

$0 \mathrm{~s}$

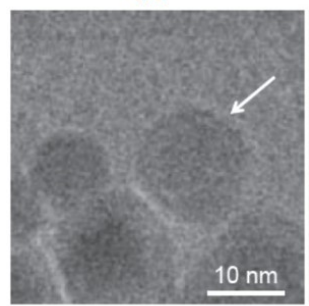

$220 \mathrm{~s}$

$540 \mathrm{~s}$

$1080 \mathrm{~s}$
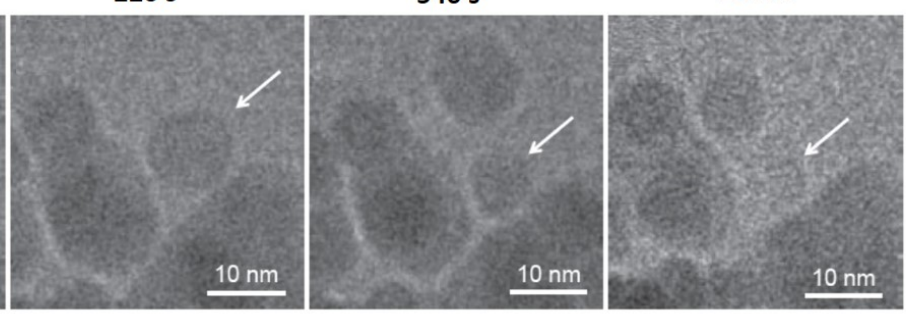

Figure 1. TEM follow-up of iron-oxide nanoparticles biodegradation. (a) In the liver of mice, iron-oxide nanoparticles are sequestered in intra-cellular lysosome and they are degraded in 3 months. This results is consistent with the decrease of the superparamagnetic signal measured at the organ level. (b) Liquid cell TEM allows visualizing the degradation of single iron-oxide nanoparticles in simple model media mimicking lysosomal environment (acidic $\mathrm{pH}+$ iron chelators). White arrows indicate the same nanoparticles from frame to frame. 